\title{
Avances en el diseño y cálculo de columnas de grava. Parte II: Grupos de columnas y consolidación secundaria
}

\section{Recent advances in the design and calculation methods of stone columns. Part II: Groups of columns and secondary compression}

$\underline{\text { Jorge Castro }}^{(*)}$

RESUMEN

Este artículo recoge algunos aspectos teóricos recientes relativos a columnas de grava que pueden resultar útiles para su diseño y cálculo. En esta segunda parte, se recogen comentarios sobre la estimación del diámetro de las columnas, sobre grupos de columnas, y sobre la problemática específica causada por asientos de consolidación secundaria. Se muestra que el número de columnas mientras se mantenga el área de sustitución bajo la cimentación no tiene influencia en el valor del asiento y que la posición de las columnas dentro de la cimentación tiene una influencia muy pequeña. La longitud crítica de las columnas es del orden de 2 veces el ancho o diámetro de la cimentación superficial. También se muestra la menor eficacia de las columnas de grava en la reducción de asientos por consolidación secundaria. Cuando se estime que los asientos por consolidación secundaria pueden ser importantes, lo recomendable es realizar una sobrecarga.

Palabras clave: columnas de grava; asiento; consolidación secundaria; diseño; grupos de columnas; suelo blando; longitud crítica.

\section{ABSTRACT}

This paper comprises recent theoretical advances for the design and analysis of stone columns. This second part comprises some comments about the estimation of column diameter, about groups of columns and about excessive settlements induced by soil secondary compression. The number of columns, if the area replacement ratio is kept constant, has a negligible influence on the settlement reduction and the column position has a small influence on the settlement reduction. The critical column length is about twice the footing width or diameter. The paper also shows the lower effectiveness of stone columns in reducing the settlements induced by soil secondary compression. When secondary compression settlements are expected to be important, soil overconsolidation by preloading is advisable.

Keywords: stone columns; settlement; secondary compression; design; group of stone columns; soft soil; critical length.

(*) Dr. Ing. Caminos, C y P. Profesor Titular. Universidad de Cantabria, Santander (España).

Persona de contacto/Corresponding author: castrogj@unican.es (J. Castro)

ORCID: https://orcid.org/oooo-0001-5815-9995 (J. Castro)

Cómo citar este artículo/Citation: Castro, Jorge (2021). Avances en el diseño y cálculo de columnas de grava. Parte II: Grupos de columnas y consolidación secundaria. Informes de la Construcción, 73(561): e371. https://doi.org/10.3989/ic.71912

Copyright: (C) 2021 CSIC. Este es un artículo de acceso abierto distribuido bajo los términos de la licencia de uso y distribución Creative Commons Reconocimiento 4.0 Internacional (CC BY 4.0). 


\section{INTRODUCCIÓN}

Esta publicación deriva de la ponencia realizada por el autor en las $19^{\text {as }}$ Jornadas Técnicas SEMSIG-AETESS, celebradas en Madrid el día 21 de febrero de 2019. Este artículo se centra en las columnas de grava, y más concretamente en el cálculo de asientos y reducción del tiempo de consolidación. Dentro de esta temática, se consideran aspectos teóricos, pero con un enfoque práctico para que puedan resultar útiles en el diseño y cálculo de columnas de grava. Debido a su extensión, se ha divido en dos partes.

En esta segunda parte se recogen comentarios sobre grupos de columnas, en particular sobre su longitud crítica, y, por otra parte, se revisa la problemática específica causada por asientos de consolidación secundaria. De forma introductoria, se recoge información relativa al diámetro de las columnas en función del terreno y método de ejecución. En la primera parte esta publicación (1), además de un ejemplo de cálculo, se presentaron aspectos básicos sobre columnas de grava que se utilizarán también en esta segunda parte, como el factor de sustitución $\left(a_{r}\right)$ que representa el porcentaje de terreno blando sustituido o desplazado por las columnas y el factor de reducción de asientos $(\beta)$, que es el cociente entre el asiento del terreno mejorado con columnas y el asiento del terreno sin mejorar. Así, se presenta a continuación, información relativa al diámetro de las columnas (Apartado 2), comentarios sobre grupos de columnas (Apartado 3), incluyendo la influencia de la posición de las columnas, del número de columnas, su longitud crítica y el cálculo de asientos, y comentarios sobre asientos de consolidación secundaria (Apartado 4). Finalmente, se resumen las principales conclusiones.

\section{DIÁMETRO DE LAS COLUMNAS}

En la primera parte de esta publicación (1) se prefirió plantear un ejemplo de cálculo, y no de diseño, para simplificar la comparación entre las diferentes metodologías de cálculo; ya que, por ejemplo, el diámetro de las columnas era un valor dado (las columnas ya se habían ejecutado y se conocía su valor medio). No obstante, el diámetro de las columnas es un parámetro de diseño que debe elegirse "a priori". El diámetro depende del método constructivo, del equipo empleado y de la resistencia del terreno. Para determinar su valor concreto, es útil la realización de columnas de prueba. A modo de referencia, en la Tabla 1 se proporcionan valores aproximados para un vibrador concreto en suelos finos y en la Figura 1 una recopilación de diversos valores.

Tabla 1. Diámetro estimado de columnas $\left(d_{c}\right)$ por vía seca con un vibrador de $0.65 \mathrm{~cm}$ de diámetro.

\begin{tabular}{|c|c|}
\hline $\mathbf{N}_{\text {SPT }}$ & $\boldsymbol{d}_{\mathbf{c}}(\mathbf{m})$ \\
\hline$<4$ & $>1$ \\
\hline 6 & 0.85 \\
\hline 8 & 0.75 \\
\hline$>10$ & 0.65 \\
\hline
\end{tabular}

\section{GRUPOS DE COLUMNAS}

En la actualidad, además de emplearse las columnas de grava bajo cargas repartidas como un tratamiento de mejora

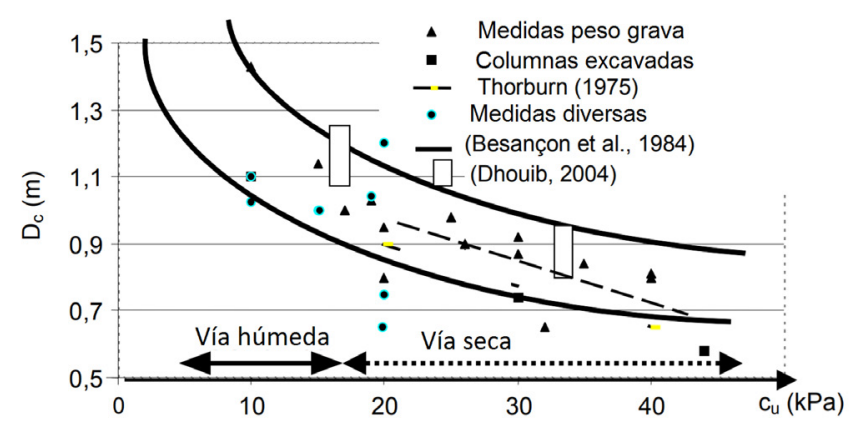

Figura 1. Diámetro de las columnas en función de la resistencia del suelo circundante (adaptado de (2)).

del terreno de carácter masivo, también se utilizan en grupos de columnas como método de cimentación bajo cargas concentradas, por ejemplo, bajo aerogeneradores, grúas portacontenedores (3) o en edificación (p. ej., (4)). En este tercer apartado se presenta un análisis de la influencia de la posición de las columnas bajo la cimentación, del número de columnas y de su longitud. El análisis se realiza de forma teórica en base a cálculos numéricos de un caso ideal e información disponible en la bibliografía de ensayos a escala reducida y cálculos numéricos (casos de estudio). Por último, se recogen unos comentarios sobre la fiabilidad del diseño de grupos de columnas.

\subsection{Influencia de la posición de las columnas}

En el diseño de losas pilotadas es necesario optimizar la posición de los pilotes dentro de la losa (p. ej., (5)); por lo que cabría plantear un proceso de optimización equivalente en el empleo de grupos de columnas de grava bajo zapatas o losas. El autor ha realizado diversos análisis por elementos finitos para estudiar cómo influye la posición de las columnas en la reducción de asientos $(6,7)$.

En la Figura 2 se muestran los resultados para un caso de referencia: una cimentación superficial de $5 \mathrm{~m}$ de lado apoyada sobre un suelo homogéneo mejorado con un grupo de 4 columnas con separaciones de entre 1 y 4 m entre ejes de columnas. Nótese que en este caso variar el espaciamiento entre columnas no significa modificar el área de sustitución, $a_{r}$ (área de las columnas entre el área de la cimentación superficial). Los asientos de la cimentación se normalizan con el asiento que tendría la misma cimentación apoyada en el mismo suelo mejorado con una única columna central con el área equivalente para mantener $a_{r}$. Se analizan tres casos: el caso en el que el comportamiento del suelo y las columnas sea elástico ("Elástico"); el caso en el que sólo existen deformaciones plásticas en las columnas ("Elasto-plástico"); y el caso en el que tanto en las columnas como en el suelo hay deformaciones plásticas ("Plástico"). En el caso estudiado se ha tomado un valor anormalmente bajo $\left(a_{r}=10 \%\right)$ para poder tener un amplio rango de variación del espaciamiento entre columnas. Se puede comprobar que la posición de las columnas tiene sólo una ligera influencia en el asiento (inferior al $6 \%$ en este caso). Además de las pequeñas diferencias, no existe una posición óptima y esta depende del caso estudiado; por ejemplo, situar las columnas cerca del borde resulta en un asiento ligeramente menor para el caso "Elástico", pero ligeramente mayor para el caso "Plástico" (Figura 2). Ello se debe a que: 
1. Cuando las columnas están cerca del borde de la cimentación, tienden a soportar una tensión vertical mayor debido a que las tensiones verticales en el borde de una cimentación rígida en arcillas son mayores.

2. Cuando las columnas están en el centro de la cimentación, están mejor confinadas lateralmente.

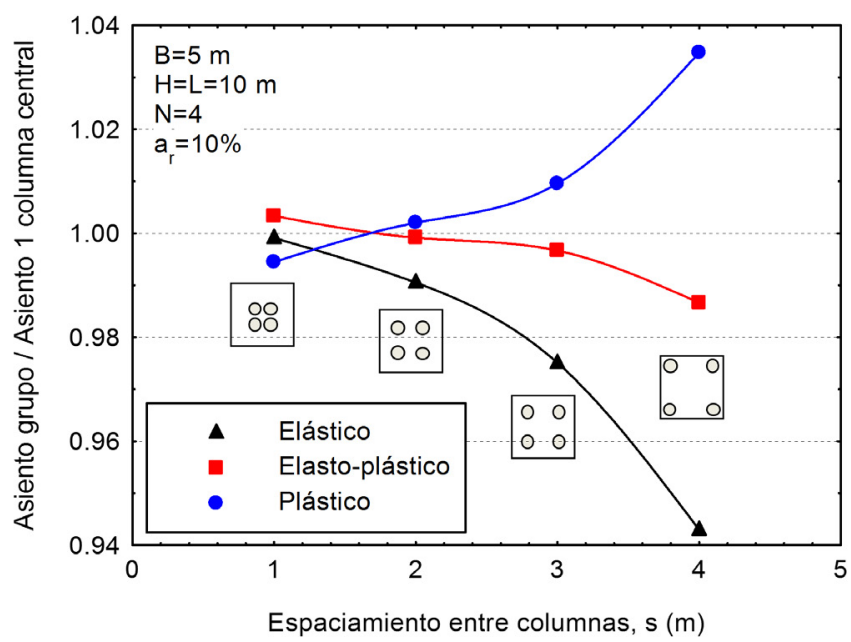

Figura 2. Variación del asiento con la posición de las columnas bajo una zapata (adaptado de (6)).

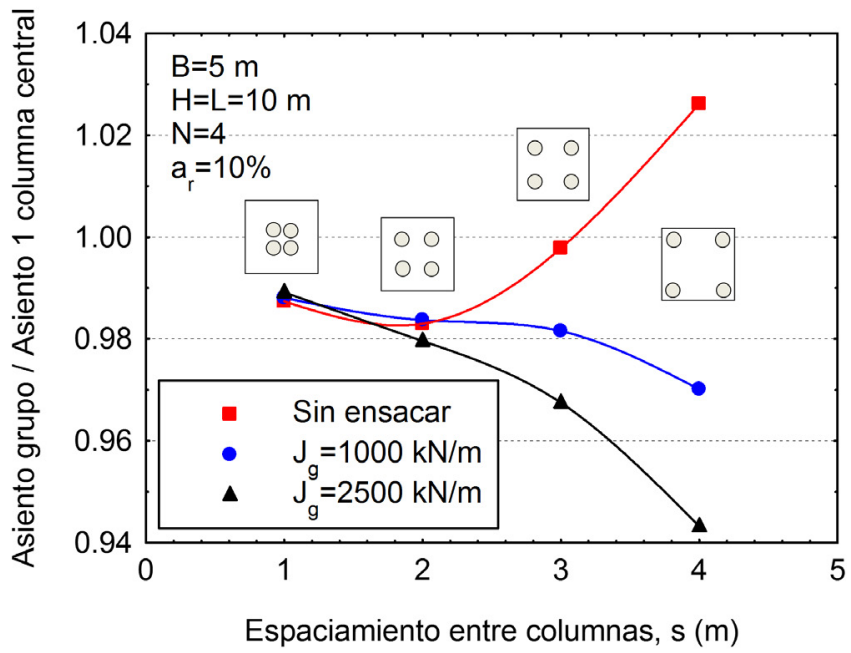

Figura 3. Variación del asiento con la posición de columnas ensacadas con geotextil bajo una zapata. Caso "Plástico". Influencia de la rigidez del geotextil (adaptado de (7)).

Es necesario destacar que, en el caso de pilotes o inclusiones rígidas, sólo el primer fenómeno es importante y no se compensa con el segundo, ya que, a diferencias de las columnas, estos elementos no dependen del confinamiento lateral proporcionado por el terreno natural.

En el caso de columnas ensacadas, se puede observar (Figura 3) que, a mayor rigidez del elemento que rodea las columnas (normalmente un geotextil) $J_{g}$, mejor confinadas están las columnas, y se reducen ligeramente los asientos cuando las columnas están cerca del borde de la cimentación superficial.

Para casos comunes, los dos efectos indicados previamente tienden a compensarse uno con el otro y por eso la posición de las columnas tiene poca influencia. Cuando las columnas están en- sacadas, además de conservarse $a_{r}$, es necesario que se conserve el factor $J_{g} / r_{c}$ para que el comportamiento sea equivalente (7).

El análisis presentado se limita al asiento final drenado, no considerando el proceso de consolidación, ni otros aspectos como los momentos flectores en la cimentación superficial, los efectos de la instalación de las columnas...

\subsection{Influencia del número de columnas}

En este apartado se comenta la influencia del número de columnas manteniendo constante el resto de parámetros, es decir, más columnas de menor diámetro o menos columnas de mayor diámetro. Este análisis no tiene una relevancia práctica directa ya que el diámetro de las columnas no es una variable que se pueda controlar fácilmente, sino que viene impuesta (ver Apartado 2 sobre diámetro de las columnas).

Algunos autores analizan la influencia de aumentar el número de columnas, pero manteniendo constante su diámetro y las dimensiones de la cimentación, lo que tiene obviamente un efecto beneficioso. Otra opción es aumentar el número de columnas manteniendo su diámetro, pero aumentando las dimensiones de la cimentación superficial para mantener constante el factor de sustitución (8). Esto último provoca un ligero incremento del confinamiento lateral con un efecto beneficioso en el factor de reducción de asientos.
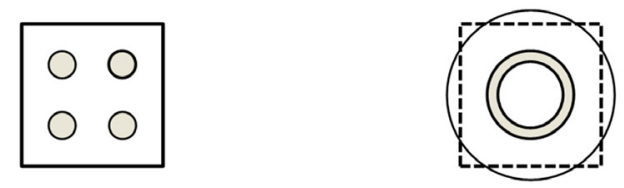

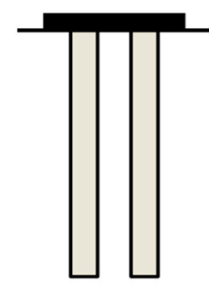

$3 \mathrm{D}$

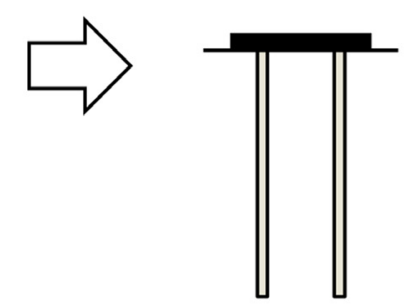

2D (Simetría axial)
Figura 4. Modelización geométrica simplificada utilizando simetría axial de un grupo de columnas.

Los análisis por elementos finitos de Castro $(6,7)$ muestran que el número de columnas como tal (manteniendo constante el resto de parámetros) no tiene influencia en la reducción de asientos y que las ligeras diferencias observadas se deben a la diferente posición relativa de las columnas bajo la cimentación. Esta influencia despreciable del número de columnas justifica la utilización de modelos simplificados en simetría axial (9). Por ejemplo, se puede sustituir un grupo de columnas (no ensacadas) bajo una cimentación superficial, por una zanja de grava de planta anular con igual área (Figura 4).

\subsection{Longitud crítica de las columnas}

Las columnas de grava pueden alcanzar un sustrato rígido ("apoyadas en la punta" / "end-bearing") o estar simplemen- 
te embebidas en un estrato blando ("flotantes" / "floating"). En este último caso, la longitud de las columnas es un parámetro de diseño, siendo algunas veces más eficaz aumentar su longitud y otras veces, añadir más columnas. De esta forma, surge el concepto de longitud crítica de las columnas. Así, columnas de mayor longitud que el valor crítico, no proporcionan una mejora apreciable. El concepto de longitud crítica es equivalente al caso de pilotes, pero los mecanismos que rigen el fenómeno son diferentes.

Una revisión de diferentes propuestas de la longitud crítica de las columnas $\left(L_{c r}\right)$ se puede ver en la Tabla $2 \mathrm{y}$, por ejemplo, en (10). Los valores presentan un cierto rango de variación y algunas veces se proporcionan en función del diámetro de la columna $\left(d_{c}\right)$, y otras en función del ancho o diámetro de la cimentación o zapata $(B, D)$. Sin embargo, se ha comprobado mediante análisis numéricos por elementos finitos $(6,7)$ que la esbeltez de la columna (cociente entre su longitud y diámetro, $L / d_{c}$ ) tiene simplemente una ligera influencia (efecto de segundo orden) en la mejora alcanzada por las columnas, y el factor $L / B$ es el más relevante. De esta forma, la longitud crítica $\left(L_{c r}\right)$ debe expresarse en función de las dimensiones de la cimentación $(B, D)(9,11)$. Por ejemplo, en los ensayos de laboratorio a escala reducida realizados por McKelvey (14), ello proporciona un valor de $L_{c r}=1.5 D$, ya que $d_{c}=25 \mathrm{~mm}, D=100$ $\mathrm{mm}, L_{c r}=150 \mathrm{~mm}$; y en Najjar et al. (16), $L_{c r}=1.7 D$, dado que $d_{c}=20 \mathrm{~mm}, D=71 \mathrm{~mm}, L_{c r}=120 \mathrm{~mm}$.

Tabla 2. Propuestas de longitud crítica de las columnas.

\begin{tabular}{|l|c|c|c|}
\hline Referencia & Tipo & $\boldsymbol{L}_{\mathbf{c r}}$ & $\boldsymbol{L}_{\mathbf{c r}}=\mathbf{f}(\boldsymbol{D})^{*}$ \\
\hline Black et al. (2011) (12) & Lab. & $8-10 d_{c}$ & $3.3-4.3 D$ \\
\hline Hughes \& Withers (1974) (13) & Lab. & $4 d_{c}$ & $4 D$ \\
\hline McKelvey (2002) (14) & Lab. & $6 d_{c}$ & $1.5 D$ \\
\hline Muir Wood et al. (2000) (15) & Lab. & $1.5 B$ & \\
\hline Najjar et al. (2010) (16) & Lab. & $6 d_{c}$ & $1.7 D$ \\
\hline Wehr (2004) (17) & Num. & $1.7 B$ & \\
\hline Zhou et al. (2017) (18) & Num. & $2 B$ & \\
\hline
\end{tabular}

Lab.: Laboratorio; Num.: Numérico

* Valores reanalizados aquí en función del tamaño de la zapata

El motivo de confusión, por el cual algunos autores expresan la longitud crítica de las columnas en función del diámetro de la columna, parece estar en la propuesta original de $\mathrm{Hu}-$ ghes y Withers (13), en la que el diámetro de la zapata y de la columna coincidían. Muir Wood et al. (15) ya señalan la importancia de las dimensiones de la cimentación y del mecanismo de deformación que se produce bajo la misma en la longitud crítica de las columnas. Castro et al. (9) muestran como para la reducción de asiento el mecanismo que controla $L_{c r}$ es el bulbo de tensiones bajo la cimentación, mientras que para la capacidad portante es el mecanismo de rotura el que es necesario considerar para analizar la longitud crítica de las columnas. De los dos mecanismos, el más profundo es el correspondiente a la reducción de asientos, por lo que $L_{c r}$ siempre suele referirse a la mejora alcanzada en cuanto a reducción de asientos.

Como la longitud crítica depende del ancho de la carga aplicada, para cargas extensas (p. ej., terraplenes), el concepto de longitud crítica no tiene sentido ya que suele ser mayor que el espesor del estrato deformable.

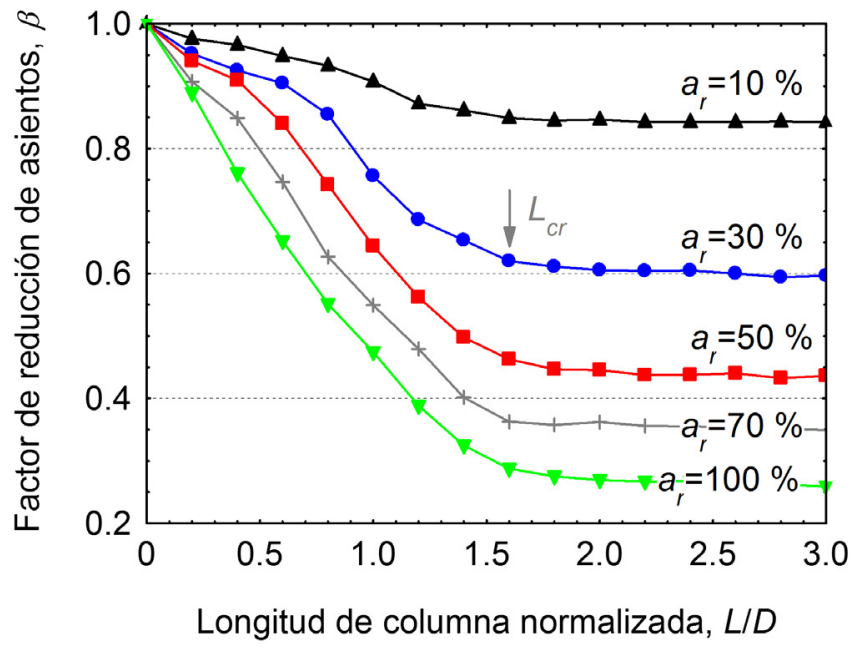

Figura 5. Longitud crítica de las columnas para diferentes valores del factor de sustitución en un caso de referencia (adaptado de (11)).

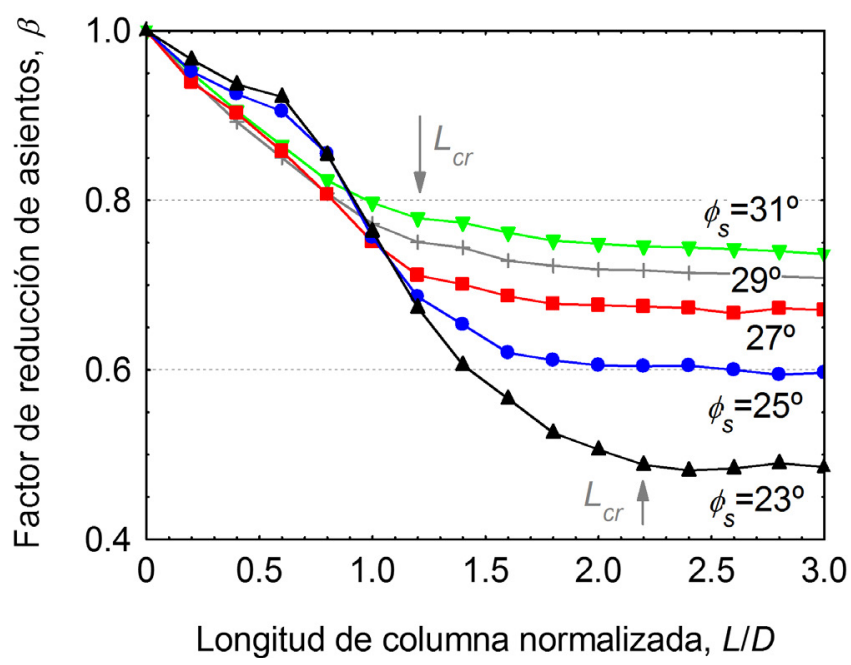

Figura 6. Variación de la longitud crítica de las columnas con la resistencia del terreno en un caso de referencia (adaptado de (11)).

Analizando la longitud crítica de las columnas mediante elementos finitos (11), se observa que esta depende del caso estudiado, pero es del orden de $L_{c r}=1.5-2 \mathrm{D}$. Su valor no varía notablemente con el área de sustitución (Figura 5), pero sí lo hace con la carga aplicada o la resistencia del terreno (Figura 6). La longitud crítica de las columnas es mayor cuanto más blando es el terreno a mejorar. Esto se debe a que la longitud crítica de las columnas para la reducción de asientos está relacionada con la aparición de deformaciones plásticas en el terreno. En el caso de columnas ensacadas, la longitud crítica es superior al de columnas ordinarias (9).

Un análisis de las deformaciones plásticas para un caso de referencia (Figura 7) muestra que se pueden distinguir 4 zonas con la profundidad: (i) una pequeña zona bajo la cimentación $(z<0.3 \cdot D)$ donde las deformaciones plásticas están restringidas por la rugosidad de la cimentación superficial; (ii) una zona $(0.3 \cdot D<Z<1.3 \cdot D)$ donde las deformaciones plásticas aparecen en el suelo que rodea las columnas por la falta de confinamiento lateral; (iii) una zona donde las deformaciones plásticas aparecen sólo en la columna (1.3 $D<Z<1.8 \cdot D)$; y (iv) una zona donde sólo existen deformaciones elásticas $(z>1.8 \cdot D)$. 
Estas 4 zonas de deformaciones plásticas se reflejan en la curva de variación de la reducción de asientos con la longitud de la columna (Figura 8): (i) el asiento se reduce sólo ligeramente porque la columna afecta solamente a la cuña de suelo rígido que se forma bajo la zapata; (ii) se produce una notable reducción del asiento porque las deformaciones plásticas se reducen tanto en la columna como en el suelo; (iii) se produce una reducción del asiento menor porque sólo se reducen las deformaciones plásticas en la punta de la columna; (iv) el asiento se reduce sólo de forma marginal porque sólo se reducen las deformaciones elásticas.

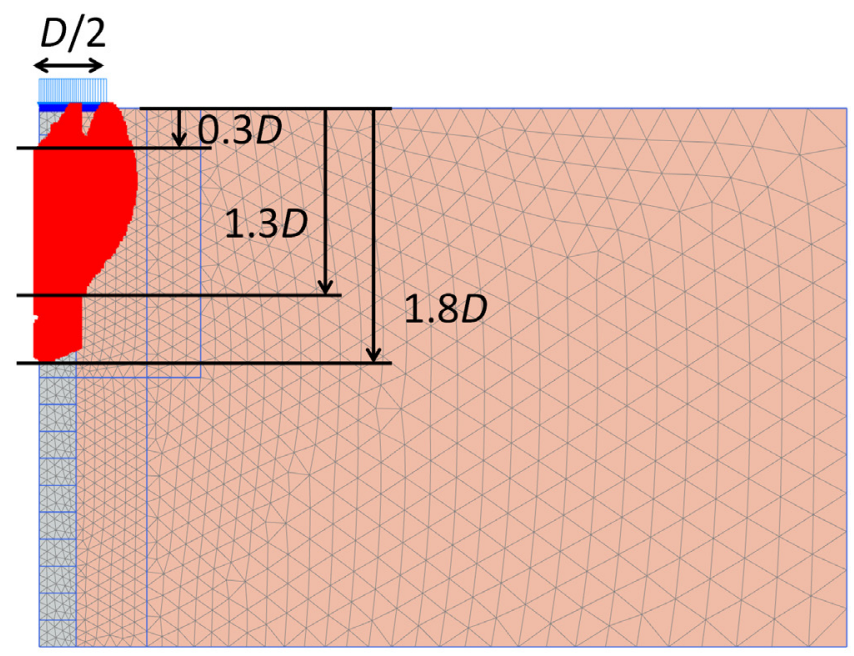

Figura 7. Puntos plásticos para un caso de referencia $\left(a_{r}=30 \%\right.$; $H=L=10 \mathrm{~m} ; D=2.5 \mathrm{~m})(11)$.

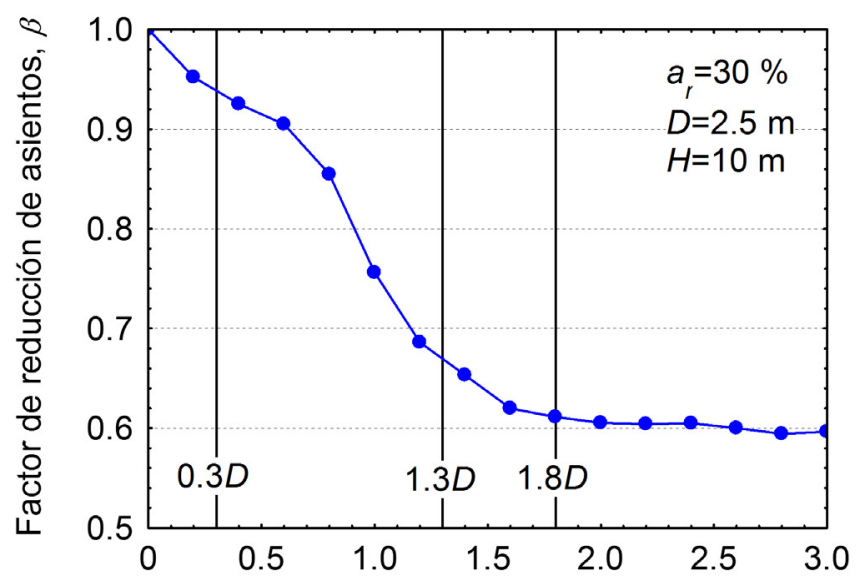

Longitud de columna normalizada, $L / D$

Figura 8. Variación de la reducción de asientos con la longitud de la columna para las diferentes zonas de deformación plástica $\left(a_{r}=30 \%\right.$; $H=10 \mathrm{~m} ; D=2.5 \mathrm{~m}$ ) (adaptado de (11)).

Estos análisis numéricos (11) se han realizado utilizando un modelo elástico lineal plástico perfecto ("modelo Mohr-Coulomb"), pero resultados similares son esperables con modelos más avanzados. La ventaja del análisis de las deformaciones plásticas presentado anteriormente es que no es necesario realizar un análisis paramétrico de la longitud de la columna y es suficiente con realizar un análisis de la extensión de las deformaciones plásticas para el caso de una columna que alcance el sustrato rígido o sea de longitud suficiente $(L>2 B)$ (Figura 9).
El análisis de la longitud crítica de las columnas presentado se ha realizado bajo la hipótesis de una única capa uniforme de terreno blando a mejorar. En el caso de que la capa no sea uniforme o de que existan capas de diferentes propiedades, es necesario tener en cuenta está situación y ajustar correspondientemente el valor de la longitud crítica.

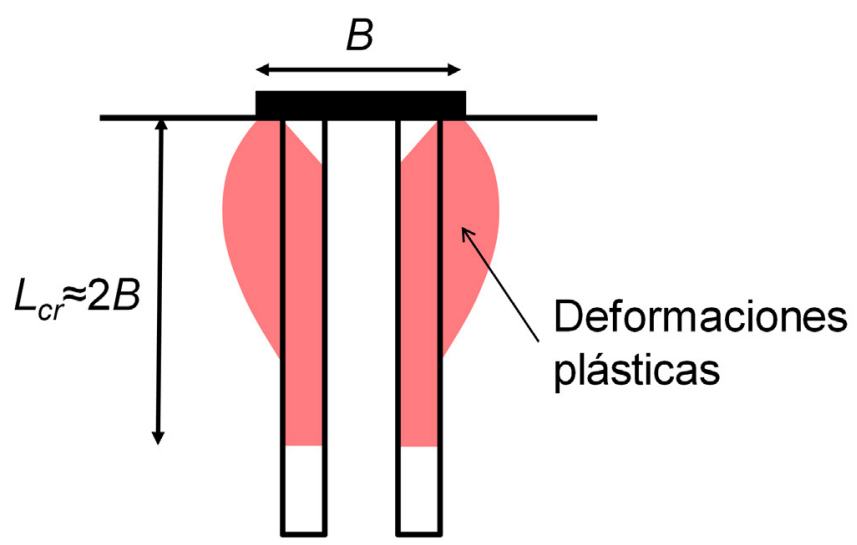

Figura 9. Justificación conceptual de la longitud crítica de las columnas como profundidad que alcanzan las deformaciones plásticas.

\subsection{Cálculo de asientos}

En la primera parte de esta publicación (1) se analizaron métodos de cálculo de asientos para aquellos casos en los que se utiliza un tratamiento con columnas de grava de carácter masivo y la simplificación geométrica de celda unidad es posible para obtener el valor del asiento máximo debido al elevado número de columnas y extensión de la carga. Sin embargo, el uso de estas formulaciones para obtener asientos en aquellos casos que se utilizan grupos de columnas provoca una sobreestimación de su valor. Esta sobreestimación se debe fundamentalmente al reparto en profundidad de la carga aplicada cuando su extensión no es elevada, de forma similar a como ocurre en terrenos no mejorados con columnas de grava.

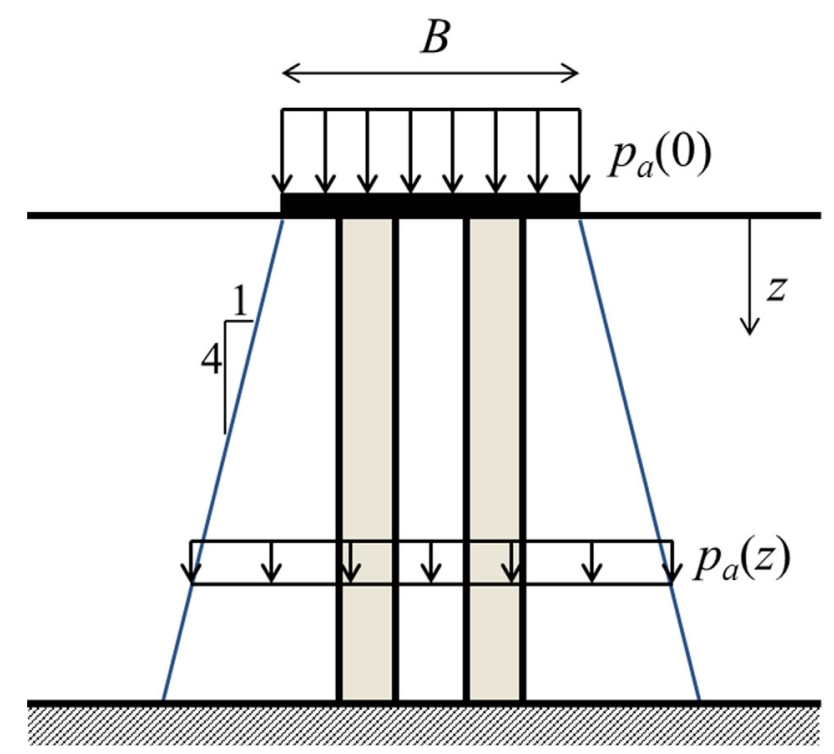

Figura 10. Distribución trapecial de la carga aplicada en profundidad. Caso con columnas de grava que alcanzan un sustrato rígido (19). 
La presencia de las columnas de grava rigidiza el terreno bajo la zapata y provoca que el reparto trapecial habitualmente considerado, 2 en vertical frente a 1 en horizontal $(2 \mathrm{~V}: 1 \mathrm{H})$, sea algo menor y se pueda considerar de forma conservadora como de 4V:1H (Figura 10) (20).

Además del reparto de la carga aplicada en profundidad, el otro aspecto que es necesario tener en cuenta es el peor confinamiento lateral de las columnas que se encuentran cerca del borde de la zapata o área cargada.

Para el caso de cimentación aislada sobre una columna de grava, Priebe (21) propuso la siguiente expresión para obtener el factor de mejora:

$$
n=\left(1-v_{s}\right) /\left(2 K_{a c}\right)
$$

Priebe (21) obtuvo la ecuación [1] usando un desarrollo analítico análogo al que él mismo había utilizado para el caso de celda unidad. Además, propuso que este valor [1] podía también considerarse válido para los casos más habituales en los que la zapata o el área cargada son mayores que el área de la columna. Por último, para estudiar cimentaciones aisladas sobre varias columnas de grava o zapatas corridas sobre columnas, propuso interpolar entre el caso de celda unidad (1) y el de columna aislada [1] (21).

En base a numerosos cálculos realizados utilizando este método, Priebe (8) propuso dos gráficos (uno para zapatas aisladas y otro para zapatas corridas o en faja) que permiten corregir el valor del asiento calculado utilizando el modelo de celda unidad (sin distribución de la carga aplicada) para obtener el asiento de las zapatas aisladas o en faja. Priebe (8) considera grupos de columnas que alcanzan un sustrato rígido (por ejemplo, Figura 10). El factor corrector depende únicamente del número de columnas y del cociente entre la profundidad y el diámetro de las columnas. El valor del factor corrector es generalmente menor de 1, pero tiende a 1 para un número de columnas muy elevado o longitud de columna muy elevada (o de forma equivalente profundidad pequeña).

Como el propio Priebe (8) indica, resulta extraño que el tamaño de la cimentación (zapata) no aparezca en estos diagramas. Sin embargo, sí que influye en los resultados y constituye un dato de entrada, aunque de forma indirecta a través del factor de sustitución y del número de columnas.

Más recientemente, Castro (19) ha desarrollado una solución analítica para el cálculo de asientos que se basa en la solución para celda unidad (22), pero incorporando las dos hipótesis siguientes para tener en cuenta los fenómenos comentados anteriormente para grupos de columnas:

- Reparto de la carga. Distribución trapecial de la carga aplicada (p.ej., Figura 10).

- Menor confinamiento lateral de las columnas. La carga en la cimentación provoca un desplazamiento radial hacia fuera de las columnas y del suelo bajo la misma. De esta forma, las columnas y el suelo bajo la zapata experimentan deformaciones radiales de extensión $\left(\varepsilon_{r}<0\right)$, mientras que el suelo lejos de la zapata se comprime radialmente $\left(\varepsilon_{r}>0\right)$. A partir de análisis numéricos, Castro
(19) observó que el punto de contacto entre deformaciones radiales positivas y negativas $\left(\varepsilon_{r}=0\right)$ coincide aproximadamente con el límite de una distribución trapecial de la carga $4 \mathrm{~V}: 1 \mathrm{H}$ (Figura 11).
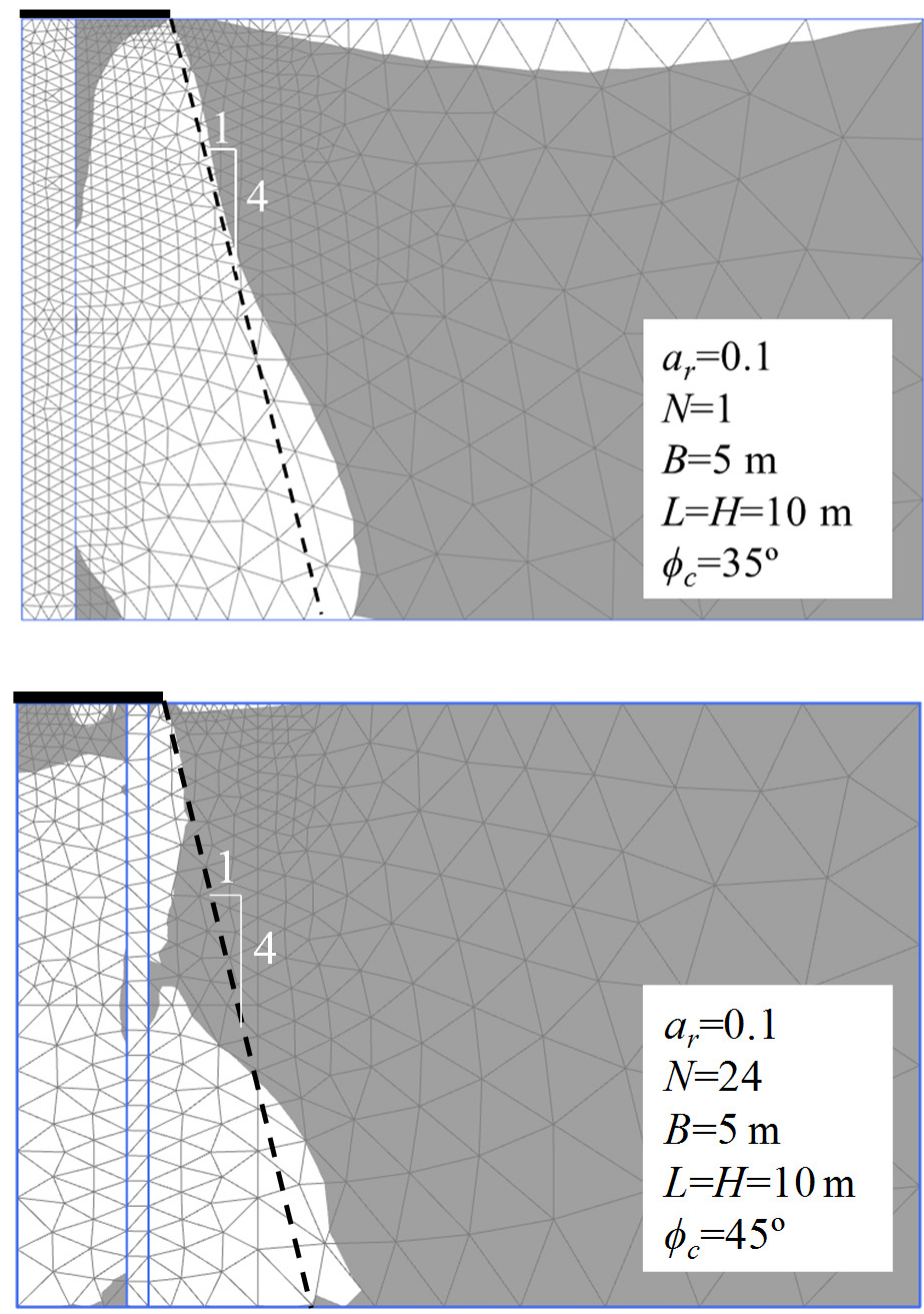

Figura 11. Límite entre deformaciones radiales de extensión $\left(\varepsilon_{r}<0\right)$ (en blanco) y de compresión ( $\varepsilon_{r}>0$ ) (en gris) (19). $N$ : número de columnas.
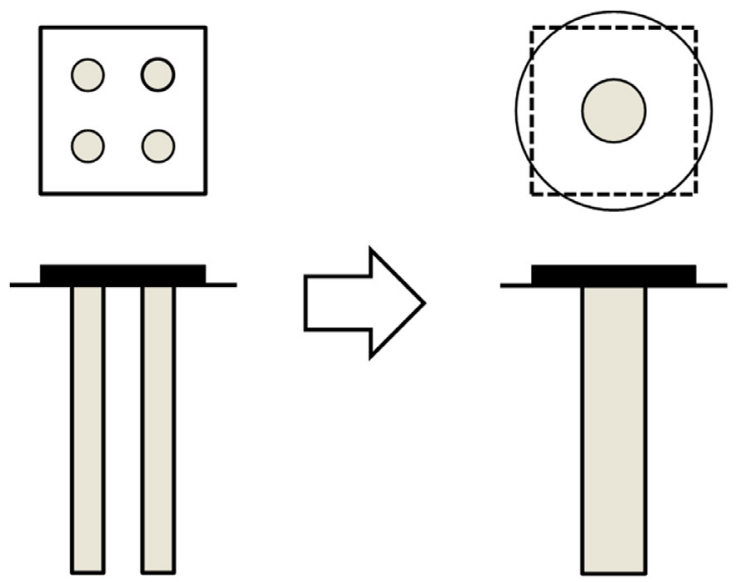

$3 \mathrm{D}$

2D (Simetría axial) Columna central equivalente

Figura 12. Modelo geométrico simplificado de columna central equivalente. 


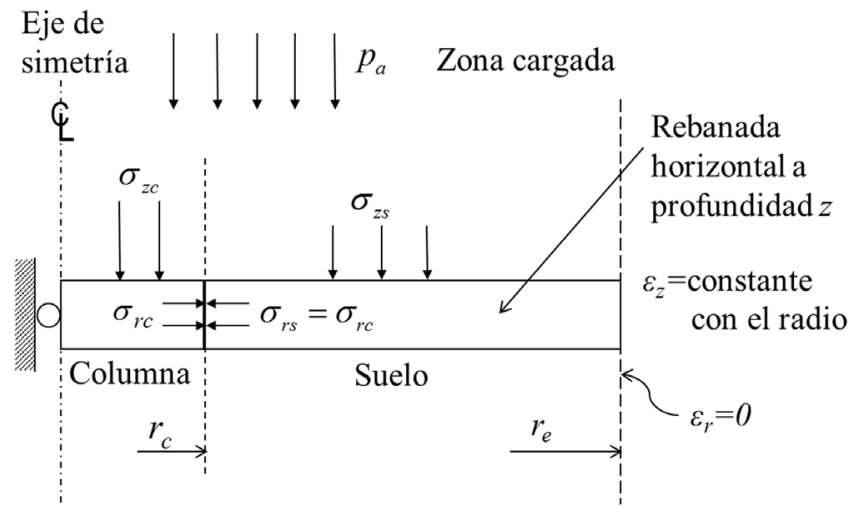

Figura 13. Rebanada de columna analizada analíticamente para obtener el valor del asiento de un grupo de columnas (adaptado de (19)).

Además, para simplificar el análisis, no se considera el proceso de consolidación (la solución es únicamente para la situación final drenada) y las diferentes columnas que aparecen bajo la zapata se convierten en una única columna central con un área equivalente (Figura 12). La validez de este modelo geométrico simplificado de una única columna central se basa en la escasa influencia de la posición y número de columnas (Apartados 3.1 y 3.2). De esta forma, el problema a estudiar analíticamente es el mostrado en la Figura 13. Su solución es análoga al caso de celda unidad con la diferencia de que la condición de contorno en el borde exterior es deformación radial nula en lugar de desplazamiento radial nulo y de que el valor de la tensión aplicada $\left(p_{a}\right)$ y la extensión del suelo circundante $\left(r_{e}\right)$ varían con la profundidad. El detalle de esta solución analítica y su utilización a través de herramientas computacionales se encuentra en (19).

\subsection{Fiabilidad en el diseño de grupos de columnas}

Alonso Pollán (23) presenta un análisis de fiabilidad de tratamientos con columnas de grava donde se muestra que las probabilidades de fallo son superiores en pequeños grupos de columnas bajo zapatas que en tratamientos de carácter masivo bajo un terraplén. De forma coloquial, se puede justificar este resultado en base a que un tratamiento de gran extensión donde se emplean un gran número de columnas lamina la variabilidad que pueda existir en algunos parámetros como, por ejemplo, el diámetro de las columnas y la resistencia al corte sin drenaje del terreno. De esta forma, para disminuir la incertidumbre en el diseño de un grupo de columnas bajo una zapata resulta recomendable (23):

- Disminuir la incertidumbre en el diámetro de la columna, mediante una correcta ejecución y control de la misma.

- Caracterizar de forma precisa la resistencia al corte sin drenaje del terreno.

- Efectuar pruebas de carga "in situ".

\section{CONSOLIDACIÓN SECUNDARIA}

En los casos anteriores sólo se ha tenido en cuenta el asiento por consolidación primaria, pero en suelos que puedan experimentar importantes asientos por consolidación secundaría, algunos autores (p. ej., (24-26)) han alertado de la menor eficacia de las columnas de grava en la reducción de estos asientos. Pugh (26) presenta dos casos en los que se superó el estado límite de servicio por asientos excesivos de losas de edificación apoyadas sobre suelos muy blandos $\left(c_{u}=8-24 \mathrm{kPa}\right)$ con presencia de materia orgánica. Los asientos con el tiempo medidos en una de ellas se muestran en la Figura 14.

Por otra parte, Sexton (24) presenta análisis por elementos finitos de la reducción de asientos proporcionada por columnas de grava para reducir asientos originados por procesos de consolidación primaria y por procesos de consolidación secundaria. Como se puede ver en la Figura 15, el factor de mejora alcanzado por las columnas es notablemente mayor en el caso de asientos por consolidación primaria que para aquellos originados por procesos de consolidación secundaria. Las curvas correspondientes a las soluciones analíticas $(22,27)$ en la Figura 15 se superponen al proporcionar valores muy similares.

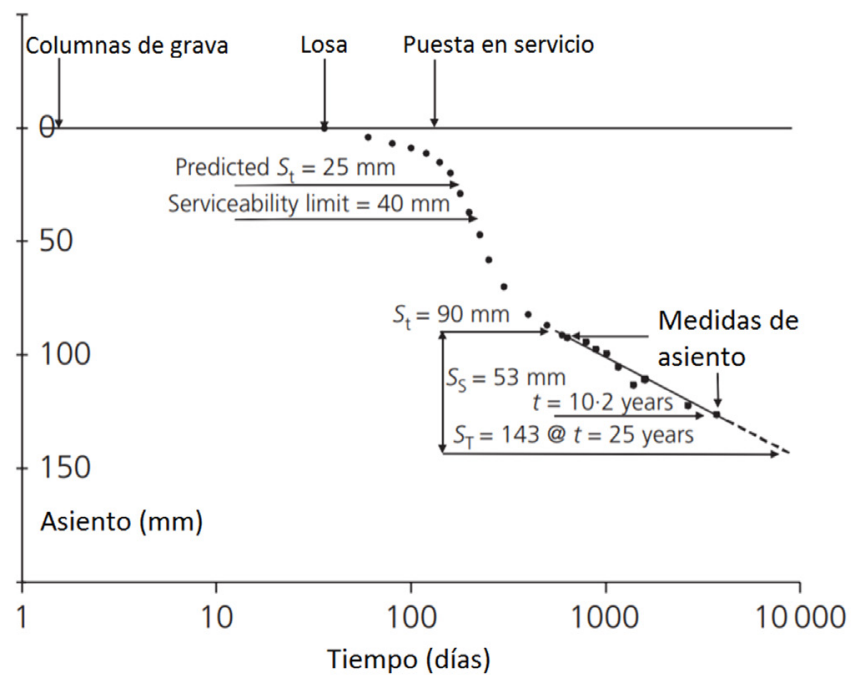

Figura 14. Problemas de asientos por consolidación secundaria en una losa cimentada sobre columnas de grava (adaptado de (26)).

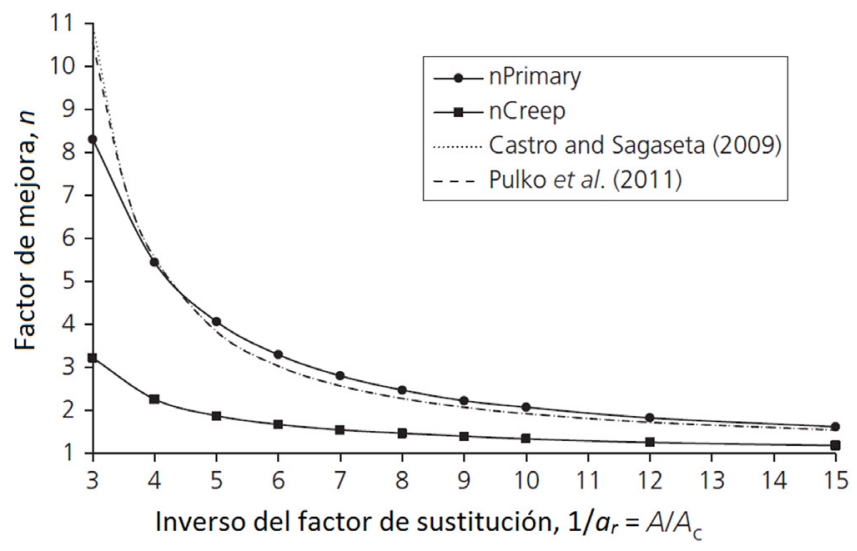

Figura 15. Factor de mejora calculado numéricamente para la reducción de asientos por consolidación primaria ("Primary") y por consolidación secundaria ("Creep") en un caso concreto (adaptado de (25)).

Desde el punto de vista del autor de esta comunicación, la menor eficacia de las columnas en la reducción de asientos por consolidación secundaria se justifica en base a los dos siguientes aspectos:

1. La naturaleza de las deformaciones por consolidación secundaria, que implican deformaciones volumétricas en el suelo blando que no corresponden a un incremento de carga.

2. Al finalizar la consolidación primaria, las columnas suelen encontrarse en un estado de plastificación triaxial, lo que 
limita notablemente que estas concentren más carga y el factor de concentración de tensiones $(S C F$, cociente entre el incremento de tensión vertical en la columna y en el suelo) aumente.

De forma general y con base empírica, Pugh (26) propone un valor conservador del factor de reducción de asientos:

$$
\beta_{\text {creep }}=1-a_{r}
$$

Este valor del factor de reducción de asientos [2] parece aplicable principalmente a los procesos de consolidación secundaria, pero es excesivamente conservador para procesos de consolidación primaria. La justificación teórica de la ecuación [2] que se propone aquí se basa en el análisis de los cambios de volumen del suelo y la columna. Si se desprecian los cambios tensionales en el suelo y la columna causados por los procesos de consolidación secundaria y se supone que la grava de la columna ha alcanzado su densidad crítica, es decir, no varía de volumen $\left(\varepsilon_{v c}=0\right)$, la mejora introducida por las columnas es únicamente la debida al porcentaje de terreno que no sufre procesos de consolidación secundaria:

$$
\begin{aligned}
\beta_{\text {creep }} & =\frac{s_{z, \text { creep }}}{s_{z 0, \text { creep }}}=\frac{\varepsilon_{v s, \text { creep }}\left(1-a_{r}\right)+\varepsilon_{v c} a_{r}}{\varepsilon_{v s, \text { creep }}}= \\
& =1-a_{r}
\end{aligned}
$$

Madhav et al. (28) propusieron una solución analítica para estudiar la reducción de asientos por procesos de consolidación secundaria que se basa en considerar la reducción de tensión vertical en el suelo debido a la carga que toma la columna mientras se produce la consolidación secundaria.

La reducción de asientos de consolidación secundaria mediante columnas de grava es actualmente objeto de investigación en el Grupo de Geotecnia de la Universidad de Cantabria en base a ensayos de laboratorio a escala reducida y desarrollo de soluciones analíticas. En cualquier caso, para reducir los asientos por consolidación secundaria cuando se estime que estos pueden ser importantes, lo recomendable es realizar una sobrecarga (p. ej., (29)).

Tradicionalmente las precargas y sobrecargas se aplican en combinación con drenes verticales, pero estas también resultan efectivas con columnas de grava (p. ej., (30,31)). Además, análisis de fiabilidad (23) muestran que, en el diseño de las columnas frente a asientos, efectuar una precarga aumenta la fiabilidad del diseño debido a la elevada influencia de la incertidumbre en la deformabilidad del suelo blando a mejorar. Estos riesgos son mayores cuando el terreno mejorado con columnas se carga con la estructura o instalación definitiva en el momento inicial (p. ej., losas de cimentación, Figura 14), frente a otros casos en los que la carga se pueda aplicar de forma gradual sin que afecte a la estructura o instalaciones definitivas (p. ej., terraplenes) (23).

\section{CONCLUSIONES}

Este artículo recoge algunos aspectos teóricos recientes relativos a columnas de grava que pueden resultar útiles para su diseño y cálculo, en particular, sobre la estimación del diámetro de las columnas, grupos de columnas y problemas derivados de asientos por consolidación secundaria.

En cuanto a grupos de columnas bajo cimentaciones superficiales, se ha mostrado que:

1. la posición de las columnas bajo la cimentación no provoca grandes diferencias en la reducción de asiento alcanzada y que la posición óptima depende de cada caso;

2. el número de columnas bajo la cimentación mientras se mantenga el área de sustitución no influye en el valor del asiento, lo que justifica la utilización de modelos geométricos simplificados como un anillo equivalente de columnas;

3. la longitud crítica de las columnas está relacionada con el desarrollo de deformaciones plásticas y es del orden de 2 veces el ancho o diámetro de la cimentación superficial para una capa uniforme de suelo blando;

4. el cálculo del asiento de un grupo de columnas se puede realizar mediante diferentes soluciones analíticas;

5. la fiabilidad en el diseño de grupos de columnas es menor que en el caso de que el tratamiento tenga un carácter masivo, por lo que se recomienda efectuar pruebas de carga "in situ".

También se han presentado algunos resultados de autores que alertan de la menor eficacia de las columnas de grava en la reducción de asientos por consolidación secundaria. En estos casos se recomienda la realización de una sobrecarga para que el terreno quede sobreconsolidado, especialmente cuando la carga se aplica una vez que la estructura o instalación es definitiva (p.ej., losas de cimentación).

\section{AGRADECIMIENTOS}

El autor agradece la invitación realizada por la Sociedad Española de Mecánica del Suelo e Ingeniería Geotécnica (SEMSIG) y por la Asociación de Empresas de la Tecnología del Suelo y Subsuelo (AETESS) para realizar una ponencia sobre columnas de grava, de la que deriva esta publicación, en las $19^{\text {as }}$ Jornadas Técnicas SEMSIG-AETESS, celebradas en Madrid el día 21 de febrero de 2019. También muestra su agradecimiento al Grupo de Geotecnia de la Universidad de Cantabria y a todas las personas y entidades que han colaborado en el estudio de las columnas de grava. Se agradece la financiación recibida por el Ministerio (BIA2009-13602, BIA2015-67479-R, MINECO/FEDER, UE).

\section{REFERENCIAS}

(1) Castro, J. (2020). Avances en el diseño y cálculo de columnas de grava. Parte I: Ejemplo de cálculo. Informes de la Construcción 72(560): e362. https://doi.org/10.3989/ic.71911.

(2) Magnan, J.P., Droniuc, N., Canepa, Y. y Dhouib, A. (2005). Réflexions sur la conception des colonnes ballastées. Proc. $16^{\text {th }}$ International Conference on Soil Mechanics and Geotechnical Engineering. Osaka. IOS Press, pp. 1377-1380.

(3) Guzmán Rojas, D. y Samper Urbano, F. (2018). Métodos de diseño para columnas de grava bajo el cimiento de grúas portacontenedores. Ingeniería Civil 192/2018, 72-85.

(4) Serridge, C.J. (2013). An evaluation of partial depth dry bottom-feed vibro stone columns to support shallow footings in deep soft clay deposits. Tesis Doctoral. Cambridge: Anglia Ruskin University. 
(5) Poulos, H.G. (2001). Piled raft foundations: design and applications. Géotechnique, 51(2): 95-113. https://doi.org/10.168o/ geot.2001.51.2.95.

(6) Castro, J. (2014). Numerical modelling of stone columns beneath a rigid footing. Computers and Geotechnics, 60: 77-87. https://doi.org/10.1016/j.compgeo.2014.03.016.

(7) Castro, J. (2017). Groups of encased stone columns: Influence of column length and arrangement. Geotextiles and Geomembranes, 45: 68-80. https://doi.org/10.1016/j.geotexmem.2016.12.001.

(8) Priebe, H.J. (1995). The design of vibro replacement. Ground Engineering, 28(10): 31-37.

(9) Castro, J., Sagaseta, C., Cañizal, J. y Da Costa, A. (2016). Modelización de columnas de grava. $10^{\circ}$ Simposio Nacional de Ingeniería Geotécnica. Sociedad Española de Mecánica del Suelo e Ingeniería Geotécnica. La Coruña, 19-21 Octubre 2016. Tomo II, pp. 567-588.

(10) Babu, M.R.D., Nayak, S. y Shivashankar, R. (2013). A critical review of construction, analysis and behaviour of stone columns. Geotechnical and Geological Engineering, 31: 1-22. https://doi.org/10.1007/s10706-012-9555-9.

(11) Castro, J., Miranda, M., Da Costa, A., Cañizal, J. y Sagaseta, C. (2019). Critical length of stone columns. Proc. $17^{\text {th }}$ Eur. Conf. Soil Mechanics and Geotechnical Engineering. 1-6 Septiembre 2019, Reykjavik, Islandia.

(12) Black, J.A., Sivakumar, V. y Bell, A. (2011). The settlement performance of stone column foundations. Géotechnique, 61(11): 909-922. https://doi.org/10.1680/geot.9.P.o14.

(13) Hughes, J.M.O. y Withers, N.J. (1974). Reinforcing of soft soils with stone columns. Ground Engineering, 7: 42-49.

(14) McKelvey, D. (2002). The performance of vibro stone column reinforced foundations in deep soft ground. Tesis Doctoral. Belfast: Queen's University of Belfast.

(15) Muir Wood, D., Hu, W. y Nash, D.F.T. (2000). Group effects in stone column foundations: model tests. Géotechnique, 50: 689-698. https://doi.org/10.1680/geot.2000.50.6.689.

(16) Najjar, S.S., Sadek, S., Maakaroun, T. (2010). Effect of sand columns on the undrained load response of soft clays. Journal of Geotechnical and Geoenvironmental Engineering, ASCE, 136(9): 1263-1277. https://doi.org/10.1061/(ASCE) GT.1943-5606.0000328.

(17) Wehr, J. (2004). Stone columns - single columns and group behaviour. $5^{\text {th }}$ Int. Conf. Ground Improvement Technologies, Kuala Lumpur, pp. 329-340.

(18) Zhou, H., Diao, Y., Zheng, G., Han, J. y Jia, R. (2017). Failure modes and bearing capacity of strip footings on soft ground reinforced by floating stone columns. Acta Geotechnica, 12: 1089-1103. https://doi.org/10.1007/s11440-017-0535-3.

(19) Castro, J. (2016). An analytical solution for the settlement of stone columns beneath rigid footings. Acta Geotechnica, 11: 309-324. https://doi.org/10.1007/s11440-014-0358-4.

(20) Sehn, A.L. y Blackburn, J.T. (2008). Predicting performance of aggregate piers. Proc. $23^{\text {rd }}$ Central Pennsylvania Geotechnical Conf., Central Pennsylvania ASCE Geotechnical Group, Hershey.

(21) Priebe, H.J. (1976). Abschatzung des Setzungsverhaltens eines dursch Stopverdichtung Verbesserten Baugrundes. Die Bautechnik 53(5), 160-162. Traducido al castellano como „Estimación de los asientos de un terreno consolidado con columnas de grava”, Boletín de información del Laboratorio de Transporte y Mecánica del Suelo n⿳0 116.

(22) Castro, J. y Sagaseta, C. (2009). Consolidation around stone columns. Influence of column deformation. International Journal for Numerical and Analytical Methods in Geomechanics, 33 (7): 851-877. https://doi.org/10.1002/nag.745.

(23) Alonso Pollán, J.A. (2014). Diseño basado en técnicas de fiabilidad del tratamiento de mejora del terreno mediante columnas de grava. Tesis Doctoral. Madrid: Universidad Politécnica de Madrid.

(24) Sexton, B.G. (2014). The influence of creep on the settlement of foundations supported by stone columns. Tesis Doctoral. Galway: National University of Ireland, Galway.

(25) Sexton, B.G. y McCabe, B.A. (2016). Stone column effectiveness in soils with creep: a numerical study. Geomechanics and Geoengineering 11 (4), 252-269. https://doi.org/10.1080/17486025.2016.1151556.

(26) Pugh, R.S. (2017). Settlement of floor slabs on stone columns in very soft clays. Proc. ICE - Geotechnical Engineering, 170: 16-26. https://doi.org/10.1680/jgeen.15.00150.

(27) Pulko, B., Majes, B. y Logar, J. (2011). Geosynthetic-encased stone columns: Analytical calculation model. Geotextiles and Geomembranes, 29: 29-39. https://doi.org/10.1016/j.geotexmem.2010.06.005.

(28) Madhav, M., Suresh, K. y Peter, E. (2010). Effect of creep on settlement of granular pile reinforced ground. International Journal of Geotechnical Engineering, 4 (4): 495-505. https://doi.org/10.3328/IJGE.2010.04.04.495-505.

(29) Alonso, E.E., Gens, A. y Lloret, A. (2000). Precompression design for secondary settlement reduction. Géotechnique, 50 (6): 645-656. https://doi.org/10.1680/geot.2000.50.6.645.

(30) Bhusan, K., Dhingra, A., Scheyhing, C. y Zhai, E. (2004). Ground improvement by stone columns and surcharge at a tank site. Proc. 5th Int. Conf. Case Histories in Geotechnical Engineering, artículo 8.36. New York, 13-17 Abril 2004.

(31) Wiltafsky, C. y Thurner, R. (2008). Soil improvement by vibro replacement and preloading for the foundation of a shopping centre on weak marine deposits. Proc. 2nd Int. Workshop on the Geotechnics of Soft Soils - Focus on Ground Improvement. Leiden: CRC Press. pp. 429-434. 